Nervenarzt $2011 \cdot 82: 1523-1524$

DOI 10.1007/s00115-011-3285-8

Online publiziert: 16. November 2011

(c) Springer-Verlag 2011

\author{
B. Widder \\ Klinik für Neurologie und Neurologische Rehabilitation, Bezirkskrankenhaus Günzburg
}

\title{
Neurologisch-psychiatrische Begutachtung
}

In allen Rechtsgebieten stehen ärztliche Gutachten an entscheidender Stelle für die Anerkennung von Renten- und Entschädigungszahlungen. Trotz der oft ausgeprägten persönlichen und sozialen Konsequenzen für die Betroffenen kommt der Begutachtung in der ärztlichen Weiter- und Fortbildung jedoch nur geringe Bedeutung zu. Mit Inkrafttreten der Weiterbildungsordnung (WBO) 2004 ist diese sogar in den meisten Bundesländern weitgehend aus dem Weiterbildungskatalog verschwunden. Vergleichbar der medizinischen Versorgung sollte aber jeder Versicherte oder Geschädigte auch Anspruch auf eine sachgerechte Begutachtung haben. Für das vorliegende Themenheft wurden 5 häufige gutachterliche Fragestellungen ausgewählt, bei denen objektive Befunde besonders schwer zu erheben sind und/oder die Klärung des Zusammenhangs regelmäßig Anlass zu umfassenden Überlegungen gibt.

Angesichts der zahlreich bestehenden Kontroversen war das „HWS-Schleudertrauma - Mythen und Fakten“ Leitthema bei der 12. Jahrestagung der Deutschen Gesellschaft für Neurowissenschaftliche Begutachtung (DGNB) im Jahr 2010 in Köln. Der Beitrag von Peter Marx gibt einen Überblick über die dort berichteten, den aktuellen Stand der Forschung wiedergebenden Ergebnisse zu den biomechanischen Grundlagen, den möglichen strukturellen Verletzungen und funktionellen Schäden nach Halswirbelsäulen(HWS)-Beschleunigungsverletzungen sowie zur Wertigkeit der verschiedenen bildgebenden Untersuchungsmethoden.

\section{( Der „Primärschaden” muss im „Vollbeweis" nachgewiesen sein}

Dreh- und Angelpunkt der Begutachtung - insbesondere leichter - SchädelHirn-Traumen ist der in allen Rechtsgebieten im „Vollbeweis“ zu führende Nachweis einer Hirnschädigung. Claus W. Wallesch, federführender Autor der diesbezüglichen Leitlinie, und Roger Schmidt nennen die hierfür erforderlichen Kriterien, die leider nicht selten aufgrund unzureichender Dokumentation der Erstversorgung nur schwer zu belegen sind. Diskutiert werden auch die Schwierigkeiten bei der Bewertung von Störungen exekutiver Funktionen nach Frontalhirnverletzungen, die neuropsychologisch kaum zu objektivieren sind und bei denen einer subtilen Fremdanamnese besondere Bedeutung zukommt.

In seinem Beitrag zur Begutachtung nach zerebralen Ischämien und Blutungen beschäftigt sich Rolf Schneider mit den häufig schwierigen Zusammenhangsfragen, wenn z. B. nach einem relativ geringfügigen Trauma eine zerebrale Ischämie, bedingt durch eine Dissektion der hirnversorgenden Arterien, auftritt. Aber auch bei intrakraniellen Blutungen ist nicht selten die schwierige Frage zu klären, ob diese traumatisch verursacht sind oder ob sie spontan oder im Gefolge einer bestehenden blutgerinnungshemmenden Medikation auftraten. Hingewiesen wird auch auf gutachtlich zu klärende Behandlungsfehler, die mit der Entwicklung der Schlaganfallbehandlung zunehmend häufiger geltend gemacht werden.
Die Begutachtung posttraumatischer Schwindelzustände wurde lange Zeit in erheblichem Umfang von HNO-ärztlichen „neurootologischen“ Einschätzungen dominiert. Es ist hier Frank Thömke und Marianne Dieterich zu verdanken, dass sie sich von neurologischer Seite dieses Themas angenommen haben. Neben Kriterienlisten, welche konkreten Befunde für den Nachweis verschiedener vestibulärer Schädigungen erforderlich sind, finden sich klare Statements zur Beurteilung geltend gemachter Schwindelsymptome z. B. im Gefolge leichterer HWS-Beschleunigungsverletzungen ohne Nachweis einer vestibulären Läsion, bei denen von HNO-ärztlicher Seite nicht selten ein unfallbedingter „Zervikalnystagmus“ oder eine „Hirnstammtaumeligkeit“ diagnostiziert wird.

Nach den zuvor überwiegend neurologisch geprägten Arbeiten beschäftigt sich der letzte Beitrag von Klaus Foerster und Bernhard Widder mit der Zusammenhangsbegutachtung geltend gemachter psychischer Unfallfolgen. Insbesondere posttraumatische Belastungsstörungen werden in jüngster Zeit in recht inflationärem Umfang von Ärzten und Psychologen diagnostiziert. Aber auch hierbei gilt die gutachtliche Grundregel, dass der psychische „Primärschaden“ - identisch wie ein körperlicher Schaden - ohne vernünftigen Zweifel („Vollbeweis“) nachgewiesen sein muss. Insbesondere bei atypischen Verläufen ist darüber hinaus nach möglichen konkurrierenden Faktoren zu fahnden.

Es bleibt zu hoffen, dass die verschiedenen Beiträge des Themenhefts insbe- 
sondere auch bei jüngeren Kolleginnen und Kollegen das Interesse an gutachterlichen Fragen verstärken und deutlich machen, dass es sich hierbei um einen spannenden Teilaspekt der klinischen Medizin handelt.

\section{B. nisow}

Prof. Dr. Dr. B. Widder

\section{Korrespondenzadresse}

Prof. Dr. Dr. B. Widder

Klinik für Neurologie und Neurologische Rehabilitation, Bezirkskrankenhaus Günzburg Ludwig-Heilmyer-Straße 2, 89312 Günzburg bernhard.widder@bkh-guenzburg.de

Interessenkonflikt. Der korrespondierende Autor gibt an, dass kein Interessenkonflikt besteht.
Der Nervenarzt bietet jeden Monat umfassende und aktuelle Beiträge zu interessanten Themenschwerpunkten aus allen Bereichen der Psychiatrie und Neurologie.

Wir haben die Jahrgänge 2010/2011 im Überblick für Sie zusammengestellt.

Möchten Sie ein bereits erschienenes Heft nachbestellen? Einzelne Ausgaben können Sie direkt bei unserem Kundenservice zum Preis von je EUR 35,- zzgl. Versandkosten beziehen:

\begin{tabular}{|c|c|c|}
\hline Der Nervenarzt & \multicolumn{2}{|l|}{2010} \\
\hline 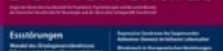 & - Heft 1/10 & Bildgebung in der Psychiatrie \\
\hline & - Heft 3/10 & Freie Themen \\
\hline & - Heft 4/10 & Freie Themen \\
\hline & - Heft 5/10 & Bipolare affektive Erkrankungen \\
\hline & - Heft 6/10 & Tiefe Hirnstimulation \\
\hline & - Heft 7/10 & S3-Leitlinie Demenzen \\
\hline & - Heft 8/10 & Neuroonkologie \\
\hline & - Heft 9/10 & S3-Leitlinie Depression \\
\hline De & - Heft 10/10 & Innovative Bildgebung beim \\
\hline 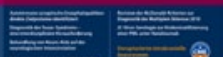 & & Parkinson-Syndorm \\
\hline 23 & - Heft 12/10 & Funktionelle Bildgebung in der Neurologie \\
\hline & 2011 & \\
\hline & - Heft 1/11 & Persönlichkeitsstörungen \\
\hline & - Heft 2/11 & Herz und Hirn \\
\hline & - Heft 3/11 & Zwangsstörungen \\
\hline er 1 & - Heft 4/11 & Freie Themen \\
\hline 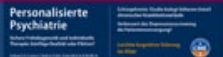 & - Heft 5/11 & Autismus \\
\hline & - Hett 6/11 & Neuromuskuläre Erkrankungen \\
\hline & - Heft 7/11 & Freie Themen \\
\hline & - Heft 8/11 & Therapie neurologischer Erkrankungen \\
\hline & - Heft 9/11 & Essstörungen \\
\hline - & - Heft 10/11 & Freie Themen \\
\hline & - Heft 11/11 & Personalisierte Psychiatrie \\
\hline & - Heft 12/11 & Neurologische-psychiatrische Begutachtung \\
\hline
\end{tabular}

So erreichen Sie unseren Kundenservice:

Springer Customer Service Center GmbH

Kundenservice Zeitschriften

Haberstr. 7

69126 Heidelberg

Tel.: +49 6221 345-4303

Fax: +49 6221 345-4229

E-Mail: leserservice@springer.com

www.DerNervenarzt.de 